\title{
EDITORIAL
}

\section{Prescription Drug Samples: Making Decisions with Imperfect Data}

\author{
Jeff Whittle, MD, MPH', and Chester B. Good, MD, MPH $H^{3,4,5}$ \\ ${ }^{1}$ Clement J. Zablocki VA Medical Center, Milwaukee, WI, USA; ${ }^{2}$ Department of Medicine, Division of General Internal Medicine, Medical \\ College of Wisconsin, Milwaukee, WI, USA; ${ }^{3}$ Section of General Internal Medicine, VA Pittsburgh Healthcare System, Pittsburgh, PA, USA; \\ ${ }^{4}$ Division of General Internal Medicine, Department of Medicine, University of Pittsburgh, Pittsburgh, PA, USA; ${ }^{5}$ Center for Health Equity \\ Research and Promotion, VA Pittsburgh Healthcare System, Pittsburgh, PA, USA.
}

$\mathrm{J}$ Gen Intern Med 23(6):890-2

DOI: $10.1007 / \mathrm{s} 11606-008-0642-9$

(c) Society of General Internal Medicine 2008

\section{INTRODUCTION}

Americans spend a large amount of money on the purchase of prescription drugs. Under Medicare's prescription drug benefit (Medicare Part D), Medicare beneficiaries must pay $\$ 4,050$ out of pocket, not including monthly premiums averaging around \$30/month (from Medicare Prescription Drug Plan Finder website), before entering the catastrophic coverage range, after which they are responsible for just 5\% of drug costs. Within the infamous doughnut hole between $\$ 2,510$ and $\$ 4,050$, beneficiaries will pay $100 \%$ of the cost of their prescription drugs. Small wonder, then, that patients, and the physicians caring for them, are grateful for access to free drug samples. These samples can enable therapeutic trials of necessary drugs, avoiding the expense of paying for a full course of a drug that might have to be discarded after the first dose leads to a side effect. A frequent justification provided by physicians is that use of free samples provides access to necessary drugs for patients who could not otherwise afford them. ${ }^{1-4}$

Unfortunately, these "free" samples do have a cost. Strong evidence links use of samples to suboptimal drug choice. ${ }^{5,6}$ Substantial administrative overhead is required to maintain an orderly sample cabinet and to ensure that only up-to-date drugs are dispensed with appropriate written instructions. ${ }^{3,7}$ Since many offices fail to invest in oversight of the sample cabinet, it is likely that many patients receive drugs with inadequate information regarding dosing schedule, side effects, and potential drug interactions. And these samples come with an expectation of access to physician and staff time by sales representatives for the companies that supply the drugs.

Thus, it is not surprising that policy analysts are examining whether the costs of having access to these samples outweigh their putative benefits. Questions regarding use of free prescription drug samples have been added to some of the most useful survey databases, including the Medicare Current Beneficiary Survey (MCBS) and the Medical Expenditure Panel Survey (MEPS). These surveys can be used to generate estimates applicable to the overall US population and many important subgroups. Research using these data has begun to

Published online May 7, 2008 be published, including an analysis of 2004 data from the MCBS by Tija et al. in the present issue of the Journal. ${ }^{8}$ Elsewhere, Cutrona et al. ${ }^{9}$ and Alexander et al. ${ }^{10}$ have presented results based on 2004 data from the MEPS. Together, these studies give an important window to the epidemiology of free sample use, which previously has been described only in limited populations.

The study by Tjia et al. is an important contribution. First, it focuses on the Medicare population. The frequency of chronic disease in older individuals makes them particularly vulnerable to medication costs. ${ }^{11}$ These individuals may also be more susceptible to the type of drug-adverse effects that provide one rationale for using free samples to initiate a new course of therapy. Moreover, this type of research has great policy relevance, since the structure of this federal program can be modified, either administratively or legislatively, in response to new research information. Since the question was put in place in 2004, changes in sample use following the advent of Medicare Part D in 2006 can be examined. Second, the MCBS and MEPS use a different question to determine whether or not a patient has received free samples. Whereas the MEPS asks respondents about sample receipt during a roughly 4-month period, the MCBS asks respondents whether they have "... ever, sometimes or never asked for or received free samples from your doctor...” While both surveys have detailed demographic information, the MCBS also asks about cost-related nonadherence to medications, a behavior that health-care providers presumably hope to minimize with samples.

The work of Tjia et al. confirms other analyses that have shown that samples are not targeted to people with lower income. Indeed, there is actually an inverse relationship between income and likelihood of accessing free samples, although this gradient is not significant after adjusting for other demographic data, having a pharmacy benefit, and comorbidity. Non-Hispanic Whites are more likely to access free samples than other racial and ethnic groups, a pattern that is reminiscent of other disparities in health-care situations requiring subjective judgments. However, the fact that free samples are not well targeted does not mean that they are not an important source of drugs for persons with limited resources-it simply means that many samples go to persons who have adequate resources.

Other studies, by Cutrona et al. and Alexander et al., come to similar conclusions regarding patterns of use: free samples are not targeted to those in greatest need. Cutrona concludes that this lack of targeting confirms that free samples serve "as 
a marketing tool, not as a safety net." While samples may be part of a marketing plan, we believe these surveys do not permit such clear conclusions. Indeed, Alexander et al.'s examination of prescription expenditures during the periods before and after drug sampling is consistent with the idea that these samples are disproportionately used by patients who have increasing drug expenditures. Again, it is not possible to determine if the sampling causes the increased drug costs by inducing use of excessively costly drugs or is a response to that increased burden. In Alexander's analysis, the vast majority of persons who received a sample drug did not in fact fill a prescription for that drug during the months following the sample.

An important new finding from Tjia's study is the strong association between accessing free samples and cost-related medication nonadherence (CRN). This finding will likely be interpreted to indicate that samples are being used to avoid this reason for nonadherence. However, as the authors note, it is also plausible that the use of free samples either causes, or is at least symptomatic of, the use of expensive drugs when less expensive ones would be as effective. Thus, the use of free samples may cause the CRN, rather than attenuate it.

How should physicians, health-care organizations, and policy makers react to these data? It is clear that they do not give a definitive answer regarding the question of whether the benefits of having a free sample cabinet outweigh the costs. However, just as one cannot wait for definitive randomized trials when treating medical illnesses, evidence-based practice dictates that decisions should be made with the data at hand. These decisions should give great weight to the systematic evidence that samples induce the use of expensive drugs when less expensive ones would work as well or better. They should consider the acknowledged dangers of dispensing drugs without appropriate labeling regarding dosing and appropriate counseling regarding specifics of use and potential side effects.

It seems clear that in some cases, particularly in large organizations such as many academic primary care practices, the sample process should be eliminated or at least extensively reworked. Many health-care organizations have taken this bold step, and we applaud them. We believe this action provides important benefits to trainees, encouraging the use of evidence-based medication choices rather than simply selecting from what is available in the sample cabinet today and role modeling a healthy separation of clinical practice and pharmaceutical sales representatives. Moreover, these large organizations can systematize the process of using the patient assistance programs provided by most pharmaceutical companies to assist those patients whose needs cannot be met by their income or insurance coverage.

Smaller practices may find it harder to abandon the sample cabinet. In addition to the (debatable) benefits for patients unable to purchase medications, the convenience of being able to initiate drug therapy without an immediate trip to the pharmacy is likely valued by many patients. ${ }^{12}$ If there is no compelling evidence that the use of free samples is bad medicine, then the lure of getting quick and "free" treatment may be a competitive advantage for those physicians who choose to continue to use samples. There is good anecdotal evidence for this; indeed a recent AARP Bulletin article ${ }^{13}$ advises that if drug costs remain high despite part $\mathrm{D}$, one might “... try asking your doctor for free samples.” However, any practice that elects to use samples should institute a formal plan for their management, including appropriate written instructions for use, checking for expiration dates and drug interactions prior to dispensing, and counseling of the patient regarding potential side effects. Most importantly, the physician should not let the samples available dictate what is prescribed. The presence of free samples must not interfere with the practice of evidence-based medicine.

On a policy level, it is appropriate to seek additional data. Although descriptive epidemiology of the type presented by Tjia et al is essential, it does not answer questions about the net and individual effects of using drug samples. This will require patient level data regarding the appropriateness and cost of the drug sampled versus alternatives, physician and patient motives for sample use, and potential and actual adverse effects of such dispensing. This type of detailed observational study may be more useful and feasible than randomized trials, which would identify net benefits, but might not capture either individual patients who benefit from the samples or system level harms. This is a non-trivial issue-the pharmaceutical industry provides $\$ 16$ billion in free samples per year, as noted by Tjia et al. The evidence that this expense improves healthcare processes or outcomes is currently limited to anecdote. Indeed, these dollars may well discourage the use of evidencebased medicine.

If, however, there are many individual patients who benefit from the free sample system, even while it increases overall medical costs and discourages evidence-based practice, then new approaches should seek to preserve these benefits while addressing the harms. Since it seems unlikely that the goal of universal affordable access to needed pharmaceuticals will be met anytime soon, partial solutions should not be shunned. Some authors have advocated the substitution of free vouchers that would allow free samples to be dispensed with the same pharmacist oversight that paid prescriptions receive. ${ }^{7}$ The pharmaceutical industry has developed formal Pharmacy Assistance Programs that provide drugs worth billions of dollars to persons with limited resources (www.pparx.org). If technology can allow these programs to be as accessible as an unlocked, poorly organized sample closet, we may be able to improve both access to drugs and evidence-based prescribing.

Corresponding Author: Jeff Whittle, MD, MPH; Clement J. Zablocki VA Medical Center, Mailstop OO (PC), 5000 W National Avenue, Milwaukee, WI 53295, USA (e-mail: jeffrey.whittle@va.gov).

\section{REFERENCES}

1. Alexander GC, Casalino LP, Meltzer Do. Physician strategies to reduce patients' out-of-pocket prescription costs. Arch Intern Med. 2005;165: 633-6.

2. Wolf BL. Drug samples: benefit or bait? (letter). JAMA. 1998;279:16989

3. Backer EL, Lebsack JA, Van Tonder RJ, Crabtree BF. The value of pharmaceutical representative visits and medication samples in community-based family practices. J Fam Pract. 2000;49:811-6.

4. Hall KB, Tett SE, Nissen LM. Perceptions of the influence of prescription medicine samples on prescribing by family physicians. Med Care. 2006;44:383-7.

5. Adair RF, Holmgren LR. Do drug samples influence resident prescribing behavior? A randomized trial. Am J Med. 2005;118:881-4. 
6. Boltri JM, Gordon ER, Vogel RL. Effect of antihypertensive samples on physician prescribing patterns. Fam Med. 2002;34:729-31.

7. Hansen LB, Saseen JJ, Westfall JM, Holcomb S, Nuzum DS, Pace WD. Evaluating sample medications in primary care: a practice-based research network study. Joint Comm J Qual \& Patient Saf. 2006;32: 68892.

8. Tjia J, Briesacher B, Soumerai SB, et al. Medicare beneficiaries and free prescription drug samples: A national survey. J Gen Intern Med. 2008; xx: yyy-zzz.

9. Cutrona SL, Woolhandler S, Lasser KE, Bor DH, McCormick D, Himmelstein DU. Characteristics of recipients of free prescription drug samples: a nationally representative analysis. Am J Pub Health. 2008;98:284-289.

10. Alexander GC, Zhang J, Basu A. Characteristics of patients receiving pharmaceutical samples and association between sample receipt and out-of-pocket prescription costs. Med Care. 2008;46:394-402.

11. Gandhi TK, Weingart SN, Borus $\mathbf{J}$, et al. Adverse drug events in ambulatory care. N Engl J Med. 2003;348:1556-64.

12. Rabin R. Free Drug Samples? Bad Idea, Some Say. The New York Times Company. 2007.

13. Barry P. Medicare Part D: In and Out of the Doughnut Hole. AARP Bulletin Online. 2006. 